\title{
Capability of Semiconducting NiO Films in Gamma Radiation Dosimetry
}

\author{
M. Guziewicz ${ }^{a}$, W. Jung ${ }^{a}$, J. Grochowski ${ }^{a}$, M. Borysiewicz $^{a}$, K. Golaszewska ${ }^{a}$, \\ R. Kruszka ${ }^{a}$, A. Baranska ${ }^{a}$, A. Piotrowska ${ }^{a}$, B.S. Witkowski ${ }^{b}$, J.Z. Domagala ${ }^{a, b}$, \\ M. Gryzinski ${ }^{c}$, K. Tyminska ${ }^{c}$ And A. Stonert ${ }^{d}$ \\ ${ }^{a}$ Institute of Electron Technology, al. Lotników 32/46, 02-668 Warsaw, Poland \\ ${ }^{b}$ Institute of Physics, Polish Academy of Sciences, al. Lotników 32/46, 02-668 Warsaw, Poland \\ ${ }^{c}$ Institute of Atomic Energy, 05-400 Otwock-Świerk, Poland \\ ${ }^{d}$ The Andrzej Soltan Institute for Nuclear Studies, Hoża 69, 00-681 Warsaw, Poland
}

\begin{abstract}
Electrical properties of $\mathrm{RF}$ magnetron sputtered $p$-NiO films were characterized after fabrication and after gamma irradiations using ${ }^{137} \mathrm{Cs}$ and ${ }^{60} \mathrm{Co}$ sources. Electrical parameters are obtained from the Hall measurements, impedance spectroscopy and $C-V$ measurement of $n-\mathrm{Si} / p-\mathrm{NiO}$ junction diodes. The results show that resistivity of the $\mathrm{NiO}$ film is gradually increased following after sequential irradiation processes because of the decrease in holes' concentration. Hole concentration of a NiO film decreases from the original value of $4.36 \times 10^{16} \mathrm{~cm}^{-3}$ to $2.86 \times 10^{16} \mathrm{~cm}^{-3}$ after ${ }^{137} \mathrm{Cs} \gamma$ irradiation with doses of $10 \mathrm{~Gy}$. In the case of $\gamma$ irradiation from ${ }^{60} \mathrm{Co}$ source, hole concentration of the film decreases from $6.3 \times 10^{16} / \mathrm{cm}^{3}$ to $4.1 \times 10^{16} / \mathrm{cm}^{3}$ and to $2.9 \times 10^{16} / \mathrm{cm}^{3}$ after successive expositions with a dose of $20 \mathrm{~Gy}$.
\end{abstract}

PACS: 73.61.Jc, 29.40.-n, 87.53.Bn

\section{Introduction}

Nickel oxide is an interesting material due to its semitransparent, semiconducting, thermoelectric and catalytic properties [1-3]. It is characterized by a direct band gap of about $4 \mathrm{eV}$ and a possible wide range of resistivity dependent on the growth method. Some of potential applications of $\mathrm{NiO}$ films in electronic devices like gas sensors, counter electrodes, displays, LEDs or variable reflectance mirrors require well controlled electrical parameters. Moreover, $\mathrm{NiO}$ films were considered to serve as a reliable passive gamma detector, especially for application in clinical dosimetry. Some optimistic results were previously demonstrated in the papers $[4,5]$, but observed changes in the current-voltage characteristics were not clarified. To verify this application possibility some test structures like $\mathrm{NiO}$ film resistors and heterojunction diodes $n-\mathrm{Si} / p-\mathrm{NiO}$ were fabricated and next exposed to gamma radiation to disclose capability of this semiconductor in gamma sensor applications. The measurements of $\mathrm{NiO}$ properties concern film resistivity, hole concentration, mobility as well as impedance of the $p-n$ diode structures. Possible changes in $\mathrm{NiO}$ films during gamma exposition can be justified by numbers of possible collisions of $\gamma$ photons with atoms. An explanation is proposed using spectra of the absorbed energy in the film. The spectra are calculated by means of the Monte Carlo simulations.

\section{Experimental}

Thin films were deposited by RF unbalanced magnetron sputtering from 3 " $\mathrm{NiO}$ target (99.99\% purity) in $\mathrm{O}_{2} / \mathrm{Ar}$ plasma (99.9999\% purity gases) onto $n$ - $\mathrm{Si}(001)$ and quartz substrates at room temperature (RT), power
$P_{\mathrm{RF}}$ of $200 \mathrm{~W}$. Oxygen content $F_{\mathrm{O} 2}$ in the plasma is defined by pressures ratio of $p_{\mathrm{O} 2} /\left(p_{\mathrm{O} 2}+p_{\mathrm{Ar}}\right)$, where $p_{\mathrm{O} 2}$ is partial oxygen pressure, $p_{\mathrm{Ar}}$ is argon pressure. Morphology and structural properties of the films were characterized using scanning electron microscopy (SEM) and X-ray diffraction (XRD). The compositions of the films were estimated by energy dispersive analysis of X-rays (EDAX) and compared with the Rutherford backscattering spectrometry (RBS) method. Electrical parameters were obtained from the Hall measurements in van der Pauw geometry and capacitance-voltage $(C-V)$ measurements of $n-\mathrm{Si} / p$-NiO junction.

As a $n$-type Si substrate for fabrication of $n-p$ junction were used high conductive samples cut from a $4^{\prime \prime} \operatorname{Si}(001)$ wafer with resistivity of $0.005 \Omega \mathrm{cm}$ and with $5 \mu \mathrm{m}$ thick epitaxial layer doped to $n=8 \times 10^{15} \mathrm{~cm}^{-3}$. The Si samples were metallized by sputtering of $(200 \mathrm{~nm}) \mathrm{Ti}$ film on the backside and next annealed in $\mathrm{Ar}$ at $600^{\circ} \mathrm{C}$ for 5 min to form Ti-silicides ohmic contact. Such prepared substrates were deoxidized in buffered hydrofluoric acid before $\mathrm{NiO}$ deposition. A pattern of circles with $1 \mathrm{~mm}$ diameter was applied during photolithography to fabricate $n-\mathrm{Si} / p-\mathrm{NiO}$ junction diodes with $300 \mathrm{~nm}$ or $400 \mathrm{~nm}$ thick $\mathrm{NiO}$ film sputtered at room temperature. (200 nm) Au top contact layer was deposited by sputtering and lift off technique to form ohmic contact to the $\mathrm{NiO}$ films. The samples were sealed from air by deposition of $(150 \mathrm{~nm})$ $\mathrm{SiO}_{2}$ film using plasma enhanced chemical vapor deposition (PECVD) at temperature of $300^{\circ} \mathrm{C}$.

The test structures were exposed to $\gamma$ radiation using ${ }^{137} \mathrm{Ce}$ source of $660 \mathrm{keV}$ energy photons and ${ }^{60} \mathrm{Co}$ source of $1332 \mathrm{keV}$ photons. The structures were characterized using capacitance-voltage $(C-V)$ and impedance meth- 
ods. $C-V$ characteristic is taken at frequency of $10 \mathrm{kHz}$, impedance is measured at frequency range from $0.5 \mathrm{kHz}$ to $2 \mathrm{MHz}$ and results are presented in form of the Nyquist plots.

Some theoretical calculations of absorbed energy by thin film $\mathrm{NiO}$ lying on $\mathrm{Si}$ substrate during gamma irradiation were performed using the general Monte Carlo $N$-particle transport code (MCNP) Ver. 5 developed at Los Alamos National Laboratory. The code dedicated to compute neutron, photon, electron, or coupled neutron/photon/electron transport in materials is here used to calculate interactions of high energetic photons and electrons with materials. The code accounts incoherent scattering, photoelectric absorption, absorption in pair production with local emission of annihilation radiation, and bremsstrahlung. The material is defined by atomic composition of elements and mass density. Densities of typical $\mathrm{NiO}$ crystal $\left(6.72 \mathrm{~g} \mathrm{~cm}^{-3}\right)$ and Si crystal $\left(2.329 \mathrm{~g} \mathrm{~cm}^{-3}\right)$ have been applied here. The sample is stroked by $662 \mathrm{keV}$ or $1332 \mathrm{keV}$ photons emitted by a point source displaced from the sample for a distance of $3.5 \mathrm{~m}$.

\section{Results and discussion}

\subsection{NiO film properties}

Conductivity of single crystal stoichiometric $\mathrm{NiO}$ is commonly very low, but for $\mathrm{NiO}$ in form of polycrystalline film, when formation of point defects is more probable, conductivity is well measurable. Applied sputtering method allowed to produce non-stoichiometric nickel oxide due to controlled oxygen flow into the $\mathrm{O}_{2}-\mathrm{Ar}$ plasma. We observed that an increase in oxygen content in gas mixture results in decrease of film resistivity and this can be explained by increase of holes' concentration as shown in Fig. 1. This result is concurrent with the ones obtained by Hotový et al. [6]. In the case of sputtering only in Ar, the film resistance is sometimes extremely high - sheet resistance of about $4 \mathrm{G} \Omega / \mathrm{sq}$ is observed for the $\mathrm{NiO}$ film deposited at $150 \mathrm{~W}$. High resistivity of the $\mathrm{NiO}$ films is associated with low hole mobility in the range of $0.02-0.8 \mathrm{~cm}^{2} /(\mathrm{Vs})$. For sputtering in oxygen, holes' concentration reaches the level of $10^{20} \mathrm{~cm}^{-3}$ and resistivity goes to the minimum of $0.14 \Omega \mathrm{cm}$. These results are consistent with the model of $p$-type conduction in $\mathrm{NiO}$ for atomic composition at ratio $\mathrm{O} / \mathrm{Ni}$ exceeding 1 and confirmed by EDX measurements of the ratio to be $1.1<\mathrm{O} / \mathrm{Ni}<1.26$ for our $p$-type films. Comparative study of the composition on few $\mathrm{NiO}$ samples by $2 \mathrm{MeV}$ $\mathrm{He}^{+}$RBS measurement confirmed the range within an error of \pm 0.02 connected with evaluation of presence of residual carbon.

XRD study of deposited $\mathrm{NiO}$ films revealed fcc structure of polycrystalline grains with texture dependent on oxygen content in plasma and temperature - $\langle 111\rangle$ texture for deposition in Ar and $\langle 200\rangle$ texture for deposition in $\mathrm{O}_{2}$ [8]. The sizes of grains are in the range $20 \div 50 \mathrm{~nm}$. Calculation of $\mathrm{NiO}$ lattice parameter revealed lattice constant increased from $4.194 \AA$ to $4.303 \AA$ for films deposited at oxygen content in gas mixture from $9 \%$ to $50 \%$,

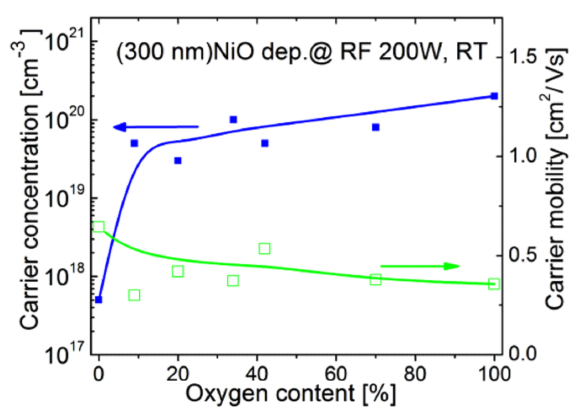

Fig. 1. Influence of the oxygen content in the gas mixture on the concentration and mobility of carriers of a $300 \mathrm{~nm}$ thick $\mathrm{NiO}$ film. The film deposited at $0 \%$ $\mathrm{O}_{2}$ content exhibited $n$-type conductivity with resultant electron concentration at a level of $5 \times 10^{17} \mathrm{~cm}^{-1}$ $( \pm 80 \%)$.

respectively. These values are larger than the lattice constant of $4.177 \AA$ characterizing bulk material. It should be mentioned that after annealing the films at $300^{\circ} \mathrm{C}$ or above, the constant is decreased.

\subsection{Influence of $\gamma$ radiation on $\mathrm{NiO}$ electrical properties}

Study on electrical properties of $p$-NiO film after $\gamma$ irradiations was performed by using both $C-V$ and impedance measurements of the $p-n$ diodes. The diode with $p$ - NiO deposited at $20 \%$ oxygen content is characterized by diffusion potential $0.64 \mathrm{~V}$ and rectifying current ratio $I_{\mathrm{ON}} / I_{\mathrm{OFF}}$ of $\approx 10^{3}$. The $C-V$ measurement allows to calculate carrier concentration of $p-\mathrm{NiO}$ based on carriers interactions at $p-n$ heterojunction. The calculation is given by Eq. (1) [7]:

$$
N_{\mathrm{a}}=n_{\mathrm{Si}} N^{*} /\left(n_{\mathrm{Si}}-N^{*}\right) \text {, }
$$

where $N_{\mathrm{a}}$ - acceptor concentration in $\mathrm{NiO}, N^{*}$ is resultant concentration of carriers near the junction interface calculated from the Mott plot obtained from $C-V$ measurements, $n_{\mathrm{Si}}$ - electron concentration in $\mathrm{Si}$. The resultant carrier concentration was measured as $N^{*}=$ $7.1 \times 10^{16} \mathrm{~cm}^{-3}$ and the acceptor concentration was calculated to be $N_{\mathrm{a}}=6.3 \times 10^{16} \mathrm{~cm}^{-3}$. Such low value of acceptor concentration is caused partially by processes of photolithography and metallization to NiO, but most likely it is a result of potential barriers on $\mathrm{NiO}$ grain boundaries and of existing traps. In a consequence we observe decrease in hole concentration measured by the $C-V$ method in this case.

The diode was exposed twice by $1332 \mathrm{keV} \gamma$ source to a dose of 20 Gy each time. As an example, a resultant carrier concentration of $N^{*}$ of the diode after irradiations is shown in Fig. 2. The resultant carrier concentration was decreased from original $7.1 \times 10^{16} \mathrm{~cm}^{-3}$ to $6.7 \times 10^{16} \mathrm{~cm}^{-3}$ and to $6.28 \times 10^{16} \mathrm{~cm}^{-3}$ for the diode irradiated for the first and second time, respectively. The hole concentration in $\mathrm{NiO}$ film was calculated to be $4.12 \times 10^{16} \mathrm{~cm}^{-3}$ and $2.9 \times 10^{16} \mathrm{~cm}^{-3}$ after the first and second irradiation, respectively. Impedance of the diode measured at 


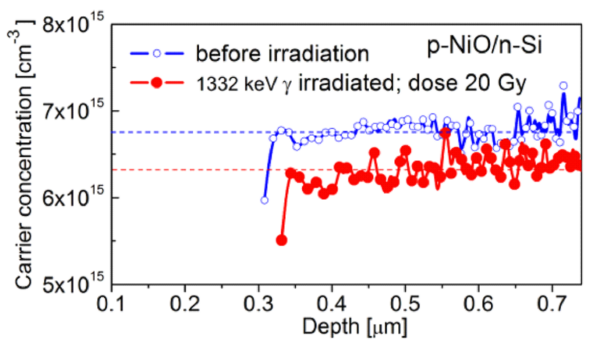

Fig. 2. The resultant carrier concentration $N^{*}$ measured at the $p-\mathrm{NiO} / n$-Si junction vs. the junction depth calculated from the $C-V$ plots for the diode before and after $\gamma$ irradiation using ${ }^{137} \mathrm{Ce}$ source.

frequency from $0.5 \mathrm{kHz}$ to $2 \mathrm{MHz}$ is presented in the form of the Nyquist plots in Fig. 3. The impedance functions $Z(\omega)=R(\omega)-\mathrm{i} X(\omega)$ are visible here as two semicircles, where this on the right side is registered at lower frequency and it is related to the junction diffusion capacitance. Symbols represent experimental data while continuous lines are simulated. The simulations involved a model for the forward bias conditions consisting of three RC impedance circuits in a series taken with the series resistance $R_{\mathrm{s}}$. Seven parameters were here engaged in the calculations and the results are shown in Fig. $3 \mathrm{~b}$ and c. Parameters $C_{1}$ and $C_{2}$ are correlated with diffusion capacitance, $C_{3}$ is associated with space charge capacitance, $R_{1}, R_{2}$ and $R_{3}$ are parallel resistances related to the $\mathrm{NiO}$ film and $R_{\mathrm{s}}$ is a series resistance. Calculated resistances of $\mathrm{NiO}$ film $R_{2}$ and $R_{3}$ are significantly increased about few times after the $\gamma$ irradiation, while capacitances of $C_{1}, C_{2}$, and $C_{3}$ remain on similar level.

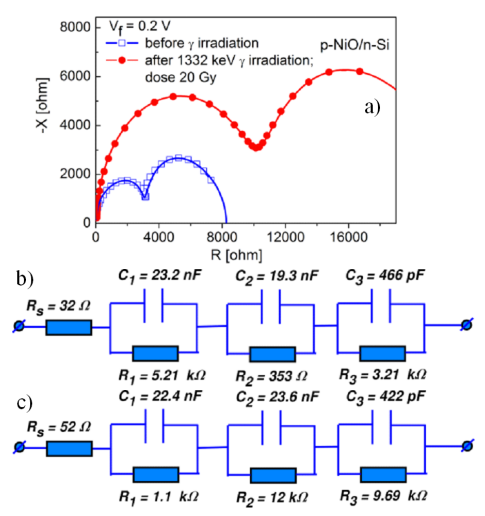

Fig. 3. (a) Nyquist plots measured at frequency from $0.5 \mathrm{kHz}$ to $2 \mathrm{MHz}$ on $p-\mathrm{NiO} / n-\mathrm{Si}$ diode $1332 \mathrm{keV} \gamma$ irradiated ones and second time with dose of 20 Gy. A model of RC circuits for the samples before (b) and after (c) the last irradiation using ${ }^{60}$ Co source.

A similar diode with $\mathrm{NiO}$ film having originally concentration $p=4.9 \times 10^{16} \mathrm{~cm}^{-3}$ was exposed to the ${ }^{137} \mathrm{Cs} \gamma$ irradiation with a dose of $18 \mathrm{~Gy}$ and characterized using similar techniques. $C-V$ measurements resulted in concentration $N_{\mathrm{a}}=3.2 \times 10^{16} \mathrm{~cm}^{-3}$ for the NiO film reveal- ing strong effect of the dose on decreasing carriers concentration. Nyquist plots from the impedance measurements are presented in Fig. 4a and following simulated results are shown in Fig. $4 \mathrm{~b}$ and c. The series resistance increased about 30 times while diffusion capacitance decreased about 16 times after exposition. Resistance of the $\mathrm{NiO}$ film is higher by one order of magnitude, while space charge capacitance has been reduced as results of the $\gamma$ exposition to dose of $18 \mathrm{~Gy}$.

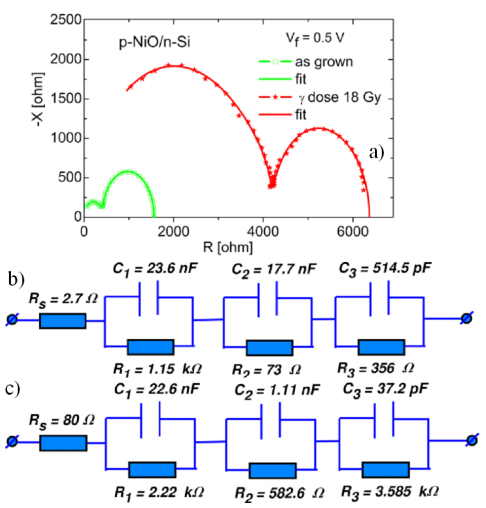

Fig. 4. (a) Nyquist plots measured at frequency from $0.5 \mathrm{kHz}$ to $2 \mathrm{MHz}$ on $p-\mathrm{NiO} / n$-Si diode before and after $662 \mathrm{keV} \gamma$ irradiation with dose of $18 \mathrm{~Gy}$ using ${ }^{137} \mathrm{Ce}$ source. A model of RC circuits for the samples before (b) and after (c) the irradiation.

This is consistent with the measurement of the Hall structures with a corresponding $\mathrm{NiO}$ film, suggesting a reduction in both concentration and mobility of holes in the film after ${ }^{137} \mathrm{Cs} \gamma$ irradiations. The results are roughly concurrent with measurements of $\mathrm{NiO}$ resistivity using $\mathrm{NiO}$ film resistors. The resistance increases successively $0.5 \div 1.5 \%$ with increase in irradiation dose of $1 \mathrm{~Gy}$. When the $p-n$ diode is irradiated sequentially with dose of 2 Gy every times, we observe that hole concentrations of a $\mathrm{NiO}$ film decreases from the original value of $4.36 \times 10^{16} \mathrm{~cm}^{-3}$ to $2.86 \times 10^{16} \mathrm{~cm}^{-3}$ after five such irradiations yielding total dose of $10 \mathrm{~Gy}$.

Our study shows that the effect of the $\gamma$ irradiation with $1332 \mathrm{keV}$ photons on hole concentration is stronger than the effect of $662 \mathrm{keV} \gamma$ irradiation at a similar dose. This higher decrease in hole concentration seems to be consistent with increased probability of multiple interactions as well as probability of electron-positron pairs creation due to sufficiently high energy.

\section{3. $M C$ simulation of $\gamma$ absorption by $\mathrm{NiO}$ film}

The mechanism responsible for observed change in electrical parameters of the $\mathrm{NiO}$ film that we propose assumes damaging $\mathrm{Ni}-\mathrm{O}$ bonds, which in turn causes destruction of $\mathrm{Ni}^{3+}$ ions responsible for hole conduction. This process lowers the number of holes and therefore changes the electrical characteristics of the $\mathrm{NiO}$ films. A basic question is if highly energetic photons are able to hinder stability of the bonds in the thin NiO film. In 
order to answer the question, the MC simulations of interaction of $662 \mathrm{keV}$ photon with the $(0.6 \mu \mathrm{m}) \mathrm{NiO}$ film were performed for the sample $(0.6 \mu \mathrm{m}) \mathrm{NiO} /(0.5 \mathrm{~mm}) \mathrm{Si}$ located in air or vacuum, as well as for the $(6 \mu \mathrm{m}) \mathrm{NiO} /$ $(0.5 \mathrm{~mm}) \mathrm{Si}$ in vacuum. As a result of simulations we get the pulse height tally which provides the energy distribution of pulses created in a cell that models a physical detector.

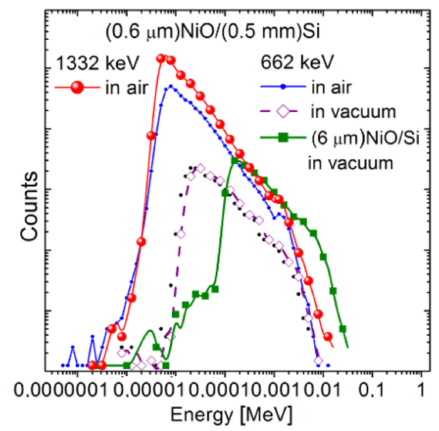

Fig. 5. Spectrum of energy absorbed in the NiO film for a MCNP detector model of $(0.6 \mu \mathrm{m}) \mathrm{NiO} /(0.5 \mathrm{~mm})$ Si displaced from the $\gamma$ source in air or in vacuum, as well as for a detector model of $(6 \mu \mathrm{m}) \mathrm{NiO} /(0.5 \mathrm{~mm}) \mathrm{Si}$ in vacuum. Spectrum is normalized to the one photon emitted by a point source of the $662 \mathrm{keV}$ or $1332 \mathrm{keV}$ photons.

The spectrum shown in Fig. 5 provides information about the energy absorbed in the NiO film. Spectrum is normalized to the one photon emitted by a point source of the $662 \mathrm{keV}$ or $1332 \mathrm{keV}$ photons. A very low counts level of $10^{-7}$ was recorded for the film situated in vacuum (not shown here), but the film placed in air is able to absorb energy strongly - even higher by a few orders of magnitude. Increased absorption in the $\mathrm{NiO}$ film is due to secondary effects of photon interactions with $\mathrm{Si}$ substrates as well as scattering processes in air, where high energy electrons are created and these strike the $\mathrm{NiO}$ film. The interaction processes influencing the $\mathrm{NiO}$ absorption cover an energy range from $0.3 \mathrm{eV}$ to $10 \mathrm{keV}$ and reveal local maxima. Other simulations for the case of $6 \mu \mathrm{m}$ thick NiO film on the Si substrate show that the absorption range is increased to $30 \mathrm{keV}$ and the position of the maximum is moved to $200 \mathrm{eV}$. In the case of $\mathrm{MC}$ simulations of interaction of $1332 \mathrm{keV}$ photon with the $(0.6 \mu \mathrm{m}) \mathrm{NiO}$ film on the $(0.5 \mathrm{~mm}) \mathrm{Si}$ sample located in air, it reveals a wider energy range of the spectra as well as maximum peak of the spectra is on the level about 3 times higher than the spectra for the $663 \mathrm{keV}$ photon. The effect is also due to electron-positron pairs creation possible at this level of photons energy. The higher possibility of interaction with $1332 \mathrm{keV}$ photon and its' absorption in the $\mathrm{NiO}$ film can explain stronger influence of the irradiation on decrease in hole concentration.

Although the MCNP V.5 does not allow an identification of the damaged $\mathrm{Ni}-\mathrm{O}$ bonds or defects created in the crystal, the spectra reveal extensive numbers of diverse energetic states which are absorbed in the thin film, some of which can cause significant damage to the band gap. In order to understand the mechanisms of radiation damages in wide band gap materials, new, more advanced models have to be designed and implemented.

\section{Conclusions}

The test diodes $p$-NiO $/ n$-Si were exposed to $\gamma$ sources and characterized using $C-V$ and impedance methods. Measurement of the diode after irradiation with $662 \mathrm{keV}$ photons for a low dose of 2 Gy showed increases in real and imaginary parts of the $\mathrm{NiO}$ impedance. This can be explained by the decrease in hole concentration. This was proved by $C-V$ measurements of the $p-n$ junction, which showed reduced hole concentration in the $\mathrm{NiO}$ film. Moreover, it was observed that $\mathrm{NiO}$ resistivity is increased after subsequent higher dose $\gamma$ irradiations. Increase in dose to 10 Gy results in stronger decrease in hole concentration from the original value of $4.9 \times 10^{16} \mathrm{~cm}^{-3}$ to $3.26 \times 10^{16} \mathrm{~cm}^{-3}$ after ${ }^{137} \mathrm{Cs} \gamma$ irradiation with doses of $18 \mathrm{~Gy}$. The effect of irradiation to similar dose (20 Gy) using $1332 \mathrm{keV}$ photons from ${ }^{60} \mathrm{Co}$ source is stronger, as hole concentration is decreased from $6.3 \times 10^{16} \mathrm{~cm}^{-3}$ to $2.9 \times 10^{16} \mathrm{~cm}^{-3}$. The changes are qualitatively justified by the Monte Carlo simulations of absorbed energy by $\mathrm{NiO}$ film exposed to gamma radiation from ${ }^{137} \mathrm{Cs}$ and ${ }^{60}$ Co sources.

Experimental results show strong influence of $\gamma$ irradiation dose on decrease in hole concentration of $\mathrm{NiO}$ and increase in real and imaginary parts of impedance of the $p-n$ diode. On the other hand increase in film resistivity observed on $\gamma$ irradiated film resistors at direct current is less pronounced. A possible application of $\mathrm{NiO}$ film as passive dosimeter requires additional optimization of film thickness, geometry of the structure as well as measurement conditions to extract better sensitivity of measured resistivity on dose unit.

\section{Acknowledgments}

The work was supported by the Polish Ministry of Science and Higher Education within the project no. 0663/ B/T02/2008/35.

\section{References}

[1] G.A. Sawatzky, J.W. Allen, Phys. Rev. Lett. 53, 2339 (1984).

[2] Z. Ying, G. Donghong, G. Yongyou, G. Fuxi, Mater. Sci. Eng B 135, 125 (2006).

[3] W. Shin, N. Murayama, Mater. Let. 45, 302 (2000).

[4] K. Arshak, O. Korostynska, F. Fahim, Sensors 3, 176 (2003).

[5] K. Arshak, O. Korostynska, D. Morris, Mater. Sci. Eng. B 118, 275 (2001).

[6] I. Hotový, J. Huran, J. Janík, A.P. Kobzev, Vacuum 51, 157 (1998).

[7] M. Guziewicz, J. Grochowski, M. Borysiewicz, E. Kaminska, J. Domagała, W. Rzodkiewicz, K. Gołaszewska, B. Witkowski, R. Kruszka, M. Ekielski, A. Piotrowska, Opt. Appl. 41, 431 (2011).

[8] S.O. Kasap, Principles of Electronic Materials and Devices, McGraw-Hill, New York 2002. 